\title{
The Connotation and Approaches of the Disciplinary Construction of Mathematics
}

\author{
Peng Wang, Lan Hu \& Qinqin Zhu \\ New Star Institute of Technology Application, Hefei City, Anhui Province, China, 230031
}

\begin{abstract}
This paper is to expound the connotation of disciplinary construction, to compare the differences between mathematics and mathematical specialties, to analyze the significance of the disciplinary construction of Mathematics, and to propose the notion of the disciplinary construction of Mathematics.
\end{abstract}

KEYWORD: university; mathematics; construction; connotation; approach

The core of higher education is talent cultivation and the disciplinary construction. The disciplinary construction is the foundation of colleges and universities teaching, scientific research as well as army service. It is also the key to promote the construction and development of colleges and universities. The construction of discipline will effectively promote the quality of talent cultivation, and it is in urgent need to solve the problem of how to construct mathematics discipline to adapt to the goal of college's construction and the need of military talented person. This paper will discuss it from the connotation of disciplinary construction, the significance of mathematics discipline in general education, and the approaches of mathematics development.

\section{CONNOTATION OF DISCIPLINARY CONSTRUCTION}

Discipline has two meanings. One is the subject and branch of the knowledge system. The difference between discipline and profession is that the former is seen from the perspective of knowledge system, while the latter refers to the social professional field. Therefore, a profession may require the integration of multiple disciplines, while a discipline can be applied in various professional fields. The other meaning of discipline refers to its relative range in college teaching and scientific and research, and the "discipline" here refers to the latter.

Mathematics discipline and mathematical specialties have different conceptual starting point and orientation. "Mathematical specialties" in the broad sense refers to the mathematics academic classification, which tends to mathematics educational "knowledge and research". "Mathematical specialties" refer to higher education training students mathematics field, which tends to the recruiting students and the nature of the job. "Mathematical specialties" refers to the academic category classified by the universities according to the military occupation division, the need of economic and military development and the classification status of the discipline development. Educational institutions make mathematics teaching plans and curriculum systems according to the cultivation objectives and plans of the mathematical specialties.

\section{SIGNIFICANCE OF DISCIPLINARY CONSTRUCTION OF MATHEMATICS}

Disciplinary construction levels determined that topranking universities would not be constructed without top-ranking disciplines in the universities construction. The advantageous universities disciplines are also the advantages of the universities, which is the significant breakthrough point of formatting school characteristics of universities. In addition, in order to educate and train top-ranking innovative talented person, universities need to be able to possess top-ranking mathematics discipline, train students with abundant mathematical knowledge and acquire rigorous mathematical thoughts. For that reason, mathematics is not only as an application tool in the universities talented person training, but it also plays an irreplaceable role in training abstract thinking and logical reasoning ability of university students.

Secondly, mathematics discipline and mathematical specialties of universities lay of 
foundation of developing military talents. Mathematics discipline formats its own culture through its distinctive mathematical language, mathematical thinking and mathematical education. This kind of discipline culture orientates and guides the development of innovative talents, and is reflected in the guidance of people's moral qualities and the improvement of logical thinking abilities. Naturally, mathematics educators in the universities should not only guide students in leaning mathematical knowledge, improving the comprehensive qualities, serving the troops better, they should also influence their students to establish correct outlooks on lie and senses of worth.

In addition, mathematics discipline plays a vital role in university education. The West Point of America points out clearly that, as a formal USA army officer, mathematical education is a necessity in the whole proceeding and sustained developing occupation career. Mathematical education focus on mastering the reasoning method, understanding the mathematical concepts, learning to use mathematical tools, so that the students fully recognize the position of mathematics in serving economics and societies of country construction.

\section{UNDERSTANDINNG AND NOTIONS OF DISCIPLINARY CONSTRUCTION OF MATHEMATICS}

\subsection{The overall plan of the disciplinary construction of Mathematics}

The education working conference of our school has discussed the five year plan through the disciplinary construction of Mathematics, which clarified the general ideas and construction objectives of mathematical disciplinary construction. In order to adapt to the requirement of the country construction and to achieve the comprehensive and rapid development of discipline, while consolidating and developing applied mathematics as an advantageous direction, it should widen the research area of mathematics discipline, unite other relevant professional disciplines, carry out the application research of intersecting disciplinary fields vigorously and apply the abundant disciplinary theoretical achievements to the reliability demonstration, evaluation of the effectiveness and the engineering practice.

\subsection{The overall objectives of the disciplinary construction of Mathematics}

The overall objectives of the disciplinary construction of Mathematics include building a reasonable structure of teaching and scientific research echelon, building a group of supervisors adapting to new training assignment needs, possessing one to two national renowned teachers and to form one to two national influential innovation groups. The objectives also include possessing one or two academic leaders who have certain domestic influence, producing high level scientific research achievements, improving the influence of discipline both domestically and internationally; building a complete functioning disciplinary laboratory with outstanding characteristics; strengthening the construction of education quality, improving the quality of training the postgraduate students and actively applying for the points of mathematical $\mathrm{PhD}$.

\subsection{The conception of the disciplinary construction of Mathematics}

First of all is to clarify the development orientation of mathematics discipline. The development of mathematics discipline requires clear orientation and orientations differ from disciplines. The orientation of mathematics discipline is related to our schools nature, purpose and objectives, and to be conjunctive with the educational characteristics, scientific orientation of our school, establish the development orientation of mathematics discipline, endeavoring to the disciplinary construction its own way, to achieve "leave some things undone in order to have some things done ', innovate the educational ideas and objectives, in order to form distinctive disciplinary advantages and win a place in the increasingly competitive higher education.

The second is to develop a plan of disciplinary construction of Mathematics with feasibility, probability and universities characteristics. The planning is the blueprint and basis of disciplinary construction. The disciplinary construction of Mathematics must be consistent with the development strategy objectives of school and to work for the achievement of the objectives. Disciplinary planning should have clear directivity, which means it concentrates the positioning structure, horizontal positioning and localization of mathematics discipline in our school. Therefore, we need to accurately orientate, clarify the research orientation, research highlights, and build feasible goal and scientific measures in making the development plans.

The third is to focus on the construction of scientific research projects and application and research work of natural scientific fund. The project is the carrier of the disciplinary construction and also a very important link in disciplinary construction. Establishing projects helps clarify the purpose and content of research as well as the key innovation. Mathematics discipline can only make great achievements and increase the overall disciplinary level with large projects and research of natural scientific fund. First of all, it needs to strengthen 
union transversely and to combine with other disciplines. The characteristics of modern science and technology are high level integration where it is unfeasible to complete large scientific research projects with mathematics discipline itself alone. In addition, it is necessary to actively encourage experts, professors and young scientific research backbones within the discipline to collide their thoughts, search for the new growth point and research new projects. In the meantime, it is necessary to focus on the construction of the base of mathematics discipline, improve the research conditions, strive for domestic high-class applied mathematics research institute, make efforts to provide ample scope and research platform for scientific and technological personnel, and create a positive working environment.

The fourth is to focus on the construction of teaching staff. The construction of the academic echelon is the key to disciplinary construction while the disciplinary construction is the core of the construction of academic staff. The disciplinary construction relies on teachers, especially the master scholars. The leaders of the disciplinary construction of Mathematics will need to have solid theoretical foundation of mathematics discipline, relatively wider scope of knowledge of relevant disciplines, strong research abilities; they also need wide fields of vision and be good at grasp the frontier of mathematics discipline, have an insight to the research opportunities and catch them, as well as be able to lead the mathematics discipline as the front of disciplinary construction. Currently, a fetal weakness of the mathematics discipline of our school is the power diffusion, which means there are no formalities of one or two particularly excellent groups and no abilities to undertake the task of large scientific research, consequently there are hardly high-class achievements and academic papers. We will have to focus on change this situation and mode, so that there will be a possibility of creating talent platform for the disciplinary construction and to remain invincible.

The last but not the least is to strengthen international communication. International communication is an important content of the disciplinary construction of mathematics, and it plays an invaluable role in the construction and development of mathematics discipline. Mathematics discipline in universities will never be truly strengthened without academic exchanges and cooperation. It would avoid detours and accelerate the development if one can actively absorb the experience of disciplinary construction of mathematics from other universities. Inviting domestic and foreign well-known university mathematics disciplinary leaders to come to our school to give a speech of introducing the frontier of disciplinary development and guide the scientific research of own discipline also promotes the rapid development of the disciplinary construction. Meanwhile, it should train their own leaders of the discipline and research, assign scientific research personnel with strong abilities to study, for example as visiting scholars, or use help from friendly universities and make breakthroughs cooperatively to speed up the training of discipline leaders and scientific research backbones.

\section{REFERENCES}

[1] Ma, Q. Try to make a comment on higher education disciplinary construction and talent cultivation, China Information Management, 2012 (6): 80-81.

[2] $\mathrm{Yu}, \mathrm{L}$. The reform of talents training model of mathematics based on market demand, 2012 (12): 23-24.

[3] Liu, J. Discussing the integration of resources, concision of direction and prompting the development of disciplinary construction of mathematics of Henan Polytechnic University, Western Science and Technology of China, 2011 (4): 93-94. 